\title{
The acid-resistance pathways of Shigella flexneri 2457T
}

\begin{abstract}
Correspondence
Naresh K. Verma

Naresh.Verma@anu.edu.au
\end{abstract}

Received 6 February 2007

Revised 3 April 2007

Accepted 2 May 2007

\author{
Amy V. Jennison and Naresh K. Verma \\ School of Biochemistry and Molecular Biology, Faculty of Science, The Australian National \\ University, Canberra, Australia
}

\begin{abstract}
The stationary-phase acid-resistance pathways of Shigella flexneri 2457T have not previously been studied. The two acid-resistance systems, the glutamate-dependent acid-resistance (GDAR) and the oxidative pathways, reported elsewhere for Escherichia coli and S. flexneri 3136, were both detected in S. flexneri 2457T. However, S. flexneri 2457T cells grown overnight under fermentative conditions and acid-shocked in minimal media in the absence of glutamate, an acid test often described as a negative control for both pathways, were capable of surviving acid challenge. It is possible that this resistance is due to the oxidative pathway operating in a non-glucose-repressible manner, or to a novel pathway present in S. flexneri 2457T. The construction of gadB and gadC mutants ruled out any contribution by the GDAR pathway, whilst further characterizing the GDAR properties of S. flexneri 2457T. Interestingly, study of the role of $r p o S$ in the oxidative pathway and the unusual acid-resistance phenotype revealed that the frameshift present in the 2457T rpoS gene results in expression of a truncated RpoS protein, which may be reduced in activity and is not essential for the acid-resistance phenotype of $S$. flexneri $2457 \mathrm{~T}$.
\end{abstract}

\section{INTRODUCTION}

Shigella flexneri causes the most communicable of bacterial diarrhoeas, shigellosis. The pathogenesis of shigellosis is dependent on the bacterium's ability to invade and colonize the gastrointestinal tract. However, in order to do so, S. flexneri must pass through the acid environment of the human stomach, which can be at $\mathrm{pH}<3$ (Giannella et al., 1972). Additionally, enteric bacteria must survive low-pH stress external to the host, such as that encountered during passage through the environment, in acidic foods and in fermenting acidified faecal material (Bhagwat \& Bhagwat, 2004; Lin et al., 1995). The ability of S. flexneri to survive these low $\mathrm{pH}$ levels appears to be an important pathogenic characteristic, and may be a contributing factor to the low infectious dose of 10-100 organisms required for shigellosis to occur (Gorden \& Small, 1993).

The in vitro test of bacterial acid resistance measures bacterial survival after $2 \mathrm{~h}$ of acid exposure at $\mathrm{pH} 2.5$, where an inoculum survival $\geqslant 10 \%$ is considered to represent resistance (Gorden \& Small, 1993). It is believed that these conditions mimic the $\mathrm{pH}$ and gastric-emptying time of a normal fasting stomach (Gorden \& Small, 1993). Bacteria able to survive this length of acid exposure are theoretically capable of breaching the gastric acid barrier to reach the more favourable environment of the intestine.

Abbreviations: AR4, acid-resistance pathway 4; GABA, gamma-aminobutyric acid; GDAR, glutamate-dependent acid resistance.
Acid resistance has been extensively studied in Escherichia coli, whilst the acid-resistance phenotype of $S$. flexneri has been described in fewer instances. Studies have shown that S. flexneri possesses at least two acid-resistance pathways that seem to be similar to two of the three pathways identified in E. coli. Acid-resistance pathway 1 (AR1) is a stationary-phase, acid-induced, glucose-repressed oxidative pathway, while acid-resistance pathway 2 (AR2) is a stationary-phase, glutamate-dependent acid-resistance (GDAR) pathway (Lin et al., 1995).

The GDAR pathway, which is induced by growth in mildly acidic conditions ( $\mathrm{pH} \mathrm{5)}$ and in fermentatively grown cells, is a glutamate decarboxylase system consisting of two homologous decarboxylase enzymes, gadA and $\operatorname{gadB}$, and an antiporter, gadC (Hersh et al., 1996; Lin et al., 1995; Waterman \& Small, 1996). This system appears to act by mopping up protons leaking into the bacterial cytosol through the decarboxylation of glutamate to gammaaminobutyric acid (GABA). GABA is then exchanged for external glutamate by the antiporter GadC, thereby maintaining the $\mathrm{pH}$ homeostasis of the cytoplasm, reversing the cell membrane potential to create an internal positive charge, and gradually alkalizing the extracellular medium (Castanie-Cornet \& Foster, 2001; Richard \& Foster, 2004). Thus, this pathway is dependent on glutamate being present in the acid-shock media (Lin et al., 1995). A number of genes involved in regulating this pathway have been identified in E. coli, revealing a complex 
network of regulation (Hommais et al., 2004; Masuda \& Church, 2003).

The GDAR pathway appears to be highly effective at acid protection in both E. coli and S. flexneri (Castanie-Cornet et al., 1999; Waterman \& Small, 1996). However, it is still unclear how important the oxidative system is to the $S$. flexneri acid-resistance phenotype, as so little is understood about the pathway. Besides some regulatory proteins, the mechanism and major components of the Shigella oxidative system are not yet known. It has been shown in E. coli that the alternative sigma factor RpoS and the cyclic AMP receptor protein (CRP) play a role in control of this pathway (Castanie-Cornet et al., 1999; Waterman \& Small, 1996). The Shigella oxidative system, characterized in strain 3136, displays similar properties to those of the oxidative pathway in E. coli, including a requirement for complex media, oxidative growth, acid induction and repression of the pathway by glucose (Lin et al., 1995). Recently, it has been shown that a fur mutant in the S. flexneri serotype 2a strain, SA100, is defective in the oxidative system. This defect appears to be due to the constitutive expression of a small regulatory RNA molecule, RyhB, in the absence of Fur regulation, repressing transcription of YdeP, an oxidoreductase that reduces acidic metabolic products in the cell (Oglesby et al., 2005).

In this study, the acid-resistance pathways of the S. flexneri serotype 2a strain $2457 \mathrm{~T}$ were studied. This strain is a highly researched Shigella strain, which has been widely used in virulence studies (Wei et al., 2003). It is not known what contribution the acid-resistance pathways of $2457 \mathrm{~T}$ make to the virulence of this strain, as they have not been previously characterized. Earlier acid-resistance studies have used S. flexneri 3136, SA100 or M25-8A (Oglesby et al., 2005; Small et al., 1994; Waterman \& Small, 2003). The known GDAR gene components are present in the $2457 \mathrm{~T}$ sequence (Wei et al., 2003). However, there is a key difference between 3136, the most studied S. flexneri strain for acid resistance, and the sequenced 2457T strain; it has been shown by Small et al. (1994) that 3136 possesses an intact rpoS gene, producing a 330 aa protein. Instead, the $2457 \mathrm{~T}$ sequence has a frameshift, $675 \mathrm{bp}$ into the rpoS sequence, creating a truncated protein of 255 aa (Wei et al., 2003). It is unclear whether this truncated RpoS retains its function as a major regulator of stationary-phase growth genes and whether this has implications for the regulation of the acid-resistance pathways of S. flexneri 2457T.

The goal of this study was to characterize the acidresistance phenotype of Shigella flexneri 2457T.

\section{METHODS}

Bacterial strains and culture conditions. The strains of $S$. flexneri and E. coli used are listed in Table 1. Minimal E salts glucose (EG) medium was prepared as described by Vogel \& Bonner (1956). The complex medium used was Luria-Bertani (LB), supplemented with $100 \mathrm{mM}$ MOPS ( $\mathrm{pH} 8$ ), MES ( $\mathrm{pH} \mathrm{5)}$ or $0.4 \%$ glucose. Nicotinic acid $\left(2 \mu \mathrm{g} \mathrm{ml}^{-1}\right)$, methionine $\left(2 \mu \mathrm{g} \mathrm{ml}^{-1}\right)$ and tryptophan $\left(2 \mu \mathrm{g} \mathrm{ml}^{-1}\right)$ were added to minimal media cultures of $S$. flexneri. Glutamate was added to EG to a final concentration of $0.012 \%$. Bacterial strains were routinely grown at $37^{\circ} \mathrm{C}$ in an orbital shaker or incubator. The media were supplemented with antibiotics as necessary. Antibiotics were used at the following concentrations: ampicillin, $10 \mu \mathrm{g} \mathrm{m}^{-1}$ and $100 \mu \mathrm{g} \mathrm{ml}^{-1}$; chloramphenicol, $30 \mu \mathrm{g} \mathrm{ml}^{-1}$.

Genetic and molecular techniques. Molecular cloning, alkalinelysis preparation of plasmid DNA and agarose gel electrophoresis were performed according to the protocols of Sambrook \& Russell (2001). DNA was purified from agarose gels using the QIAquick kit (Qiagen). Primers used for cloning complementation vectors were obtained from Sigma Proligo. PCR was performed with Pfu polymerase, as specified by the manufacturer (Promega). DNA sequencing was performed at the Biomolecular Resources Facility, John Curtin School of Medical Research (Australian National University), with the ABI 3730 capillary sequence analyser using the Big Dye version 3.1 (Applied Biosystems) sequencing protocol. The molecular weight marker SPP-1/EcoRI was used to determine DNA fragment sizes. Chromosomal DNA was prepared using the procedure described by Huan et al. (1995).

Construction of insertion mutants. Two different knockout approaches were used to produce chromosomal insertions. For construction of rpoS and gadC insertion mutants, a suicide plasmid

Table 1. Bacterial strains used in this study

\begin{tabular}{|lll|}
\hline \multicolumn{1}{|c|}{ Characteristics } & \multicolumn{1}{c|}{ Reference or source } \\
\hline S. flexneri & & \\
SFL1001 & S. flexneri wild-type 2457T, serotype 2a & Walter Reed Army Institute of Research \\
SFL1629 & SFL1001 rpoS::pNV1234 & This work \\
SFL1641 & SFL1001 gadC::pNV1206 & This work \\
SFL1647 & SFL1001 rpoS::pNV1234/pNV1314 & This work \\
SFL1648 & SFL1001 rpoS::pNV1234/pNV1315 & This work \\
SFL1650 & SFL1001 gadB::Cm & This work \\
SFL1723 & SFL1001 gadB::Cm/pNV1389 & This work \\
SFL1729 & SFL1001 gadC::pNV1206/pNV1397 & This work \\
E. coli & & \\
B1401 & MG1655 K-12 wild-type & P. R Reeves, University of Sydney \\
\hline
\end{tabular}


approach was taken. Internal fragments of 281 and $395 \mathrm{bp}$ for rpoS and gadC, respectively, were amplified by PCR using gadCSaxF (5'-CGAGCTCTTACCGTTCTGATGTCCC-3'), gadCXbaR (5'-CGTCTAGATTTCACCCCTTTACCACC-3'), rpoSF294Sac (5' -AGAGCTCCTTGCGTCTGGTGGTAAA- $3^{\prime}$ ) and rpoSR575Xba (5'-CATCTAGATCTCTTCCGCACTTGGTT- $3^{\prime}$ ). Gene fragments were cloned into the SacI/XbaI sites of pGP704 to create pNV1234 for rpoS and pNV1206 for gadC. The resulting plasmids can only replicate in hosts expressing the $\pi$ (pir) protein. Thus, when introduced into S. flexneri $2457 \mathrm{~T}$, which does not contain the pir protein, the only way to maintain the plasmid is for it to integrate into the chromosome via homologous integration. Ampicillin-resistant $\left(10 \mu \mathrm{g} \mathrm{ml}^{-1}\right)$ colonies were screened for correct integration by PCR, where integration generates a larger band. Correct chromosomal location of the integration of pNV1234 into rpoS in SFL1629 was confirmed by Western blotting, and integration of pNV1206 into gadC in SFL1641 was confirmed by Southern blotting (data not shown). The rpoS genewas amplified from S. flexneri $2457 \mathrm{~T}$ with primers 2457TRpoSSacF (5'-TGAGCTCGGCGGAACCAGGCTTTTG-3') and RpoSXbaR (5'-CATCTAGACCTGAATCTGGCGAACAC-3'), and amplified from E. coli MG1655 with MG1655SacF (5'-TGAGCTCCAAGGGATCACGGGTAGG-3') and MG1655XbaR (5'-GTTCTAGAGTTGCGTATGGGCGGTAA-3'). S. flexneri 2457 T and E. coli PCR-amplified $r p o S$ genes were cloned into $S a c I / X b a I$ sites of $\mathrm{pBC}$ SK + (Stratagene) to create pNV1314 and pNV1315, respectively. gadC was amplified from S. flexneri $2457 \mathrm{~T}$ using gadCSac $\left(5^{\prime}-\right.$ TCGAGCTCTCACTGGCATTAGCAACG-3') and gadCXba( $5^{\prime}$-GTCTAGACGCTGGTCTTCTAATCGT-3'), and cloned into SacI/XbaI sites of pBC SK + to create pNV1397.

The gadB mutant was constructed using the PCR $\lambda$ red integration approach (Datsenko \& Wanner, 2000). Primers gadBKOF (5'-GTCGCATTTCAGATTATCAATGATGAATTATATCTTGAGTGTAGGCTGGAGCTGCTTC-3') and gadBKOR (5' -GTTTCGGGTGATCGCTGAGATATTTCAGGGAGGCTTTGTACATATGAATATCCTCCTTAG- $3^{\prime}$ ) were designed, carrying $40 \mathrm{nt}$ of sequence from the $121-161$ and $1330-1360$ bp regions of the gadB gene. These primers also carry the P1 and P2 sequences, designed to amplify the cat gene from $\mathrm{pKD} 3$; the P2 sequence contains a ribosomal binding site to create non-polar knockouts. The resulting PCR product of the cat gene, containing flanking regions with $\operatorname{gadB}$ homology, was electroporated into $S$. flexneri $2457 \mathrm{~T}$ carrying the temperature-sensitive pKD46 helper plasmid. Chloramphenicol-resistant colonies were screened for integration by PCR, using the $\mathrm{cl}$ primer specific to the cat gene with a $\operatorname{gadB}$-specific primer (Datsenko \& Wanner, 2000). Correct insertion of the cat PCR fragment in SFL1650 was confirmed by PCR and sequencing (data not shown). The loss of pKD46 was tested for by antibiotic plating. The S. flexneri $2457 \mathrm{~T}$ gadB gene was amplified by the primers gadBcompF $\left(5^{\prime}\right.$-CTCTAGACACTTGCTTACTTTATCG-3') and gadBcompR (5'-TAGAGCTCCGTTGCTAATGCCAGTGAAC- $3^{\prime}$ ) and cloned into the XbaI/SacI sites of pBS KS + to create pNV1389. Table 2 shows the plasmids used in this study.

Western blotting. Strains were grown at $37{ }^{\circ} \mathrm{C}$ overnight to stationary phase in LB containing the required antibiotics. The optical densities of cultures were determined and the cell numbers were calculated from growth curves. Equal numbers of cells were collected by centrifugation and resuspended in $100 \mu \mathrm{l} 1 \times$ loading buffer [50 mM Tris- $\mathrm{HCl}(\mathrm{pH}$ 6.8), $2 \%$ SDS, $10 \%$ glycerol (v/v), $2.5 \% \beta$ mercaptoethanol, $0.1 \%$ bromophenol blue] and left at $100{ }^{\circ} \mathrm{C}$ for 5 min. Protein samples were run on a $15 \%$ SDS-polyacrylamide gel. After protein transfer by wet blotting onto PVDF membranes, RpoS was detected using rabbit anti-RpoS polyclonal sera (Ruiz \& Silhavy, 2003) and sheep anti-rabbit IgG horseradish peroxidase (ICN). The signal was developed with Supersignal West Pico chemiluminescent substrate (Pierce) and captured on Hyperfilm (Amersham).

Catalase assay. The qualitative catalase activity was estimated by dropping $10 \mu \mathrm{l} 30 \% \mathrm{H}_{2} \mathrm{O}_{2}$ onto individual colonies grown overnight on LB plates at $37^{\circ} \mathrm{C}$ (Subbarayan \& Sarkar, 2004). The rapidity and degree of bubbling were measured for each strain. Five individual colonies were tested for each strain.

Acid-resistance assays. Acid-resistance assays were carried out as described by Lin et al. (1995) and Castanie-Cornet et al. (1999). Cells were grown overnight in one of several media, including LBG (LB plus $0.4 \%$ glucose), buffered LB [100 mM MOPS (pH 8) or $100 \mathrm{mM}$ MES (pH 5)] and EG. To examine the GDAR pathway, cells were grown overnight to stationary phase at $\mathrm{pH} 5$ or 8 , in the presence or absence of oxygen, and acid-shocked at pH 2.5 in EG containing $0.012 \%$ glutamate. The $\mathrm{pH} 8$ cultures did not contain glucose, as the consequent fermentative growth lowers the $\mathrm{pH}$ of the growth medium to $\mathrm{pH} 5$ overnight. Cultures grown for $24 \mathrm{~h}$ were diluted $1: 1000$ into $5 \mathrm{ml}$ warmed pH $2.5 \mathrm{EG}$, supplemented where indicated with $0.012 \%$ glutamate, and incubated for $2 \mathrm{~h}$ at $37^{\circ} \mathrm{C}$, shaken at 140 r.p.m. Cultures grown without oxygenation were grown in $5 \mathrm{ml}$ Bijou bottles with tightly closed lids with shaking at 20 r.p.m. Viable cell counts were determined at 0 and $2 \mathrm{~h}$ post acid challenge by serial dilution and plating on LB agar plates. At least three repetitions were performed for each experiment.

Table 2. Plasmids used in this study

\begin{tabular}{|lll|}
\hline Plasmid & \multicolumn{1}{c|}{ Characteristics } & \multicolumn{1}{c|}{ Reference or source } \\
\hline pBS KS + & Cloning vector & Stratagene \\
pBC SK + & Cloning vector & Stratagene \\
pKD3 & PCR knockout template plasmid & Datsenko \& Wanner (2000) \\
pKD46 & Red recombinase expression plasmid & Datsenko \& Wanner (2000) \\
pGP704 & Suicide vector, R6K ori & Miller \& Mekalanos (1988) \\
pNV1206 & pGP704 with internal fragment of gadC & This work \\
pNV1234 & pGP704 with internal fragment of $r p o S$ & This work \\
pNV1314 & pBC SK + with SFL1001 rpoS & This work \\
pNV1315 & pBC SK + with MG1655 rpoS & This work \\
pNV1389 & pBS KS + with gadB & This work \\
pNV1397 & pBC SK + with gadC & This work \\
\hline
\end{tabular}


Table 3. Characteristics of the GDAR pathway for S. flexneri 2457T SFL1001

\begin{tabular}{|llccc|}
\hline Condition & \multicolumn{1}{c}{$\begin{array}{c}\text { Adaptation } \\
\text { medium }^{*}\end{array}$} & $\begin{array}{c}\text { pH } \mathbf{2 . 5} \text { challenge } \\
\text { medium } \dagger\end{array}$ & Percentage survival $\ddagger$ & SEM \\
\hline 1 & LBG (pH 5) WO & EG + Glt & 88.6 & 3.90 \\
2 & LBG (pH 5) & EG + Glt & 37.9 & 8.90 \\
3 & LB (pH 8) WO & EG + Glt & 3.30 & 0.39 \\
4 & LB (pH 8) & EG + Glt & $<0.001$ & 0 \\
5 & EG (pH 5) WO & EG + Glt & 52.0 & 9.90 \\
6 & EG (pH 5) & EG + Glt & 5.87 & 1.60 \\
\hline
\end{tabular}

${ }^{*}$ Adaptation involved overnight growth in media listed. Glt, glutamate added at $0.012 \%$; WO, growth without oxygenation.

†Challenge was a $1: 1000$ dilution of overnight culture into $\mathrm{pH} 2.5$ medium for $2 \mathrm{~h}$.

$¥$ Survival is stated as a percentage of the inoculum. Results are the mean \pm SEM of three experiments.

\section{RESULTS}

\section{The GDAR pathway of SFL1001}

Table 3 shows the GDAR pathway characteristics for the $S$. flexneri 2457T strain SFL1001. Acid induction was displayed by the strain at $\mathrm{pH} 5$, as there was very little acid survival detected for cultures grown at $\mathrm{pH} 8$, whilst cultures grown overnight at $\mathrm{pH} 5$ were capable of surviving acid exposure (Table 3, condition 2 vs 4). Additionally, SFL1001 exhibited increased resistance to acid exposure when grown in culture without oxygenation (Table 3, condition 1 vs 2 and condition 3 vs 4). Finally, GDAR resistance in $S$. flexneri $2457 \mathrm{~T}$ is not dependent on overnight growth in complex media, as acid resistance was detected when the strain was grown overnight in EG (pH 5) (Table 3, conditions 5 and 6). The negative control for the GDAR pathway, which was acid shock in EG (pH 2.5 ) without glutamate, is included in Table 4 and will be discussed below.

\section{The oxidative acid-resistance pathway of SFL1001}

Table 4 contains the results for the glutamate-independent oxidative acid-resistance pathway of SFL1001; cells were grown overnight to stationary phase and acid-shocked at $\mathrm{pH} 2.5$ in EG. Buffers were included in the overnight medium to maintain the exact $\mathrm{pH}$ levels. Similar to E. coli, the S. flexneri $2457 \mathrm{~T}$ oxidative system protected cells at pH 2.5 in minimal media in the absence of glutamate (Table 4, condition 1). There was a clear requirement for oxidative growth conditions, as resistance was not present in cells grown without oxygenation (Table 4, condition 1 vs condition 2). Furthermore, as observed by Lin et al. (1995) for S. flexneri 3136, the oxidative pathway of $S$. flexneri $2457 \mathrm{~T}$ required overnight growth in complex media and could not be detected in cells grown overnight in minimal media at pH 5 (Table 4, condition 4).

Transfer of cells into fresh media is sufficient to remove the inhibitor and restore acid resistance (Castanie-Cornet et al.,

Table 4. Acid resistance for the oxidative acid-resistance pathway of S. flexneri $2457 T$ strain SFL1001

\begin{tabular}{|llccc|}
\hline Condition & \multicolumn{1}{c}{ Adaptation medium $^{*}$} & $\mathbf{p H ~ 2 . 5}$ challenge medium $\boldsymbol{\dagger}^{\dagger}$ & Percentage survival $\neq$ & SEM \\
\hline 1 & LB-MES (pH 5) & EG & 54.9 & 5.80 \\
2 & LB-MES (pH 5) WO & EG & 0.53 & 0.13 \\
3 & LB-MOPS (pH 8) & EG & $<0.001$ & 0 \\
4 & EG (pH 5) & EG & $<0.001$ & 0 \\
5 & LB-MES (pH 5) + Glt & EG & 57.0 & 8.70 \\
6 & LB-MOPS (pH 8) + Glt & EG & $<0.001$ & 0 \\
7 & LB-MOPS (pH 8) +fresh EG & EG & $<0.001$ & 0 \\
8 & LBG (pH 5) & EG & 59.4 & 10.4 \\
9 & LBG (pH 5) WO & EG & $<0.01$ & 0 \\
\hline
\end{tabular}

*Adaptation involved overnight growth in media listed. WO, growth without oxygenation; Glt, $5.9 \mathrm{mM}$ glutamate added to overnight cultures immediately prior to acid challenge; fresh EG, cells were grown overnight in the media indicated before being transferred to fresh EG ( $\mathrm{pH} 7$ ).

$\dagger$ Challenge was $1: 1000$ dilution of overnight culture into $\mathrm{pH} 2.5$ medium for $2 \mathrm{~h}$.

$\ddagger$ Survival is stated as a percentage of the inoculum. Results are the mean \pm SEM of three experiments. 
1999). In this study, the oxidative pathway of the S. flexneri 2457T strain SFL1001 appeared to display acid induction: resistance was detectable when cells were grown overnight at $\mathrm{pH} 5$, but not when they were grown at $\mathrm{pH} 8$ (Table 4, condition 1 vs condition 3). To determine whether this behaviour was due to the production of an inhibitor, as reported for E. coli, cells grown overnight at $\mathrm{pH} 8$ were transferred to fresh EG ( $\mathrm{pH}$ 7) prior to acid challenge. This treatment did not generate acid resistance in these cells (Table 4, condition 7), suggesting that the lack of resistance in $\mathrm{pH}$ 8-grown cells of $S$. flexneri $2457 \mathrm{~T}$ was not due to an inhibitor, but that oxidatively grown cells require acid induction in order to display acid resistance in EG at pH 2.5. Additionally, Castanie-Cornet et al. (1999) demonstrated that the addition of $5.9 \mathrm{mM}$ glutamate to cells grown overnight at $\mathrm{pH} 8$ could activate oxidativepathway acid resistance. Table 4 , conditions 5 and 6 , shows that the addition of $5.9 \mathrm{mM}$ glutamate to SFL1001 stationary cells had no effect on the levels of acid resistance.

\section{Detection of non-glucose-repressed, glutamate- independent acid resistance in SFL1001}

Many previous studies of $S$. flexneri and E. coli have stated that the oxidative resistance pathway is in fact glucose repressed, and not observed in cells grown to stationary phase in media containing glucose (Lin et al., 1995, 1996). It should be noted that the glucose contained within the acid-shock medium EG does not appear to play a role in glucose repression, suggesting that it is stationary-phase growth in the presence of glucose that is important. In those studies, it appears that overnight growth in LBG at pH 5 suppresses the oxidative pathway, such that no protection is observed when cells are acid-shocked in EG ( $\mathrm{pH}$ 2.5). The absence of glutamate in the acid-shock media should prevent the GDAR pathway from operating, making these acid test conditions essentially a negative control for both pathways. However, as shown in Table 4, condition 8 , nearly $60 \%$ cell survival was possible for $S$. flexneri $2457 \mathrm{~T}$ under these conditions. When cells were grown similarly at $\mathrm{pH} 5$ in LBG but with limited oxygenation, which favours the GDAR system, no acid resistance was detected (Table 4, condition 9). This result seems to suggest that the response is due to the oxidative pathway, which prefers oxygenated growth and can operate in acid-shock minimal media without glutamate supplementation. If this is the case, the pathway is not repressed by the overnight fermentative growth of the cells in glucose-containing media. As S. flexneri $2457 \mathrm{~T}$ requires growth factor supplementation in minimal media, it was necessary to determine whether the presence of the supplements was contributing to this novel resistance. Similar resistance was seen when EG ( $\mathrm{pH} 2.5)$ was not supplemented with the $S$. flexneri growth supplements methionine, tryptophan or nicotinic acid, demonstrating that the resistance was not dependent on the presence of these growth supplements (data not shown). This resistance, observed in cells grown to stationary phase overnight in LBG ( $\mathrm{pH} \mathrm{5)}$ and acid-shocked in EG ( $\mathrm{pH} 2.5$ ), will be referred to as non-glucose-repressed, glutamate-independent acid resistance, or acid-resistance pathway 4 (AR4) for convenience. To determine the contribution of either the oxidative or the GDAR pathway to AR4, mutants were constructed in acid-resistance-related genes known to contribute to the GDAR and oxidative pathways of $S$. flexneri 3136.

\section{Role of RpoS in SFL1001 acid resistance}

It is believed that the oxidative acid-resistance pathway is RpoS-dependent, and RpoS appears to be important, but not essential, for operation of the GDAR pathway in $S$. flexneri (Small et al., 1994; Waterman \& Small, 1996). However, in S. flexneri 2457T, the rpoS gene contains a frameshift mutation that results in a truncated protein missing the last 89 amino acids (Fig. 1) (Wei et al., 2003). The rpoS gene of SFL1001 was sequenced to confirm the presence of the frameshift mutation in SFL1001, and aligned to the full-size rpoS gene sequence of $S$. flexneri 301 published by Small et al. (1994) (Fig. 1). (a)

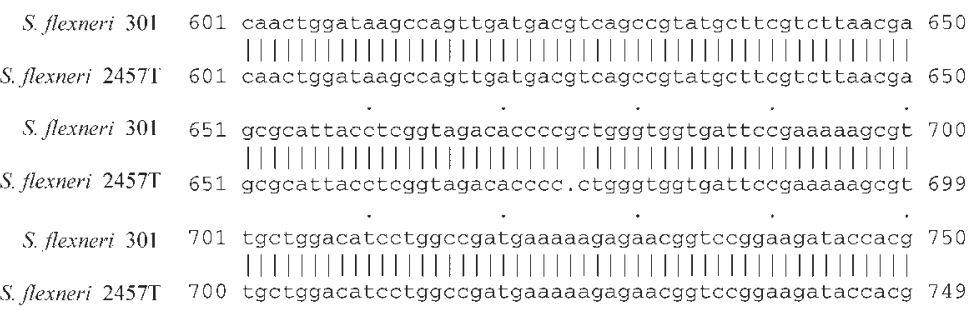

(b)

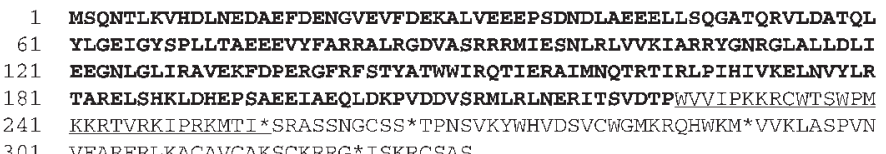

301 VFARFRLKACAVCAKSCKRRG* ISKRCSAS
Fig. 1. (a) Nucleotide sequence of the $S$. flexneri 2457T rpoS gene, sequenced from SFL1001, aligned with the published sequence of $S$. flexneri 301 rpoS (NCBI accession number P35540). The SFL1001 2457T rpoS sequence correlates with the published 2457T sequence, except that there is a missing $\mathrm{G}$ at $675 \mathrm{bp}$. (b) S. flexneri $2457 \mathrm{~T}$ RpoS predicted truncated protein sequence. The amino acids in bold type are 100\% homologous to the sequence of $S$. flexneri 301 RpoS. The frameshift results in the altered amino acids (indicated by underlined type), creating a stop codon (indicated by the first asterisk) 255 aa into the protein. 
To determine whether this altered RpoS protein was still important for the acid-resistance pathways of S. flexneri $2457 \mathrm{~T}$, in particular the non-glucose-repressed, glutamateindependent resistance, an rpoS mutant was produced for SFL1001. A Western blot was performed with whole-cell lysate to determine whether the truncated RpoS is in fact expressed by SFL1001, and also to confirm the successful disruption of rpoS in SFL1629 (Fig. 2). A truncated RpoS of approximately $30 \mathrm{kDa}$ was detected for SFL1001, which was not found in SFL1629, confirming that the gene disruption had been successful in SFL1629. The full-sized RpoS of E. coli MG1655 was observed as a $38 \mathrm{kDa}$ band. RpoS proteins of 30 and $38 \mathrm{kDa}$ were observed for the complemented strains SFL1647 and SFL1648, respectively, as expected. Although roughly the same number of cells was loaded on the 1D SDS-PAGE gel for each strain, there was a large discrepancy in the expression levels of RpoS. The plasmid-based RpoS levels were higher, as would be expected, as there are multiple copies of the gene within each cell, with expression of RpoS by SFL1647 being extremely high for unknown reasons.

Acid-resistance assays were performed on the strains grown in LB-MES ( $\mathrm{pH} 5$ ) and LBG ( $\mathrm{pH}$ 5), and challenged in EG $(\mathrm{pH} 2.5)$ or EG ( $\mathrm{pH} 2.5)$ supplemented with $0.012 \%$ glutamate (Fig. 3). The rpoS disruption did not have a significant effect on either the GDAR (condition 3, Fig. 3) or the oxidative resistance pathway (condition 2, Fig. 3), as SFL1629 did not display any attenuation in acid resistance. Likewise, there was no change in AR4 resistance (condition 1, Fig. 3). It seems that the truncated RpoS of $S$. flexneri $2457 \mathrm{~T}$ has little or no importance in the regulation of the $S$. flexneri $2457 \mathrm{~T}$ acid-resistance pathways.

\section{Activity of SFL1001 truncated RpoS measured by catalase assay}

To determine the activity of the truncated RpoS of SFL1001, it is possible to measure the activity of RpoS
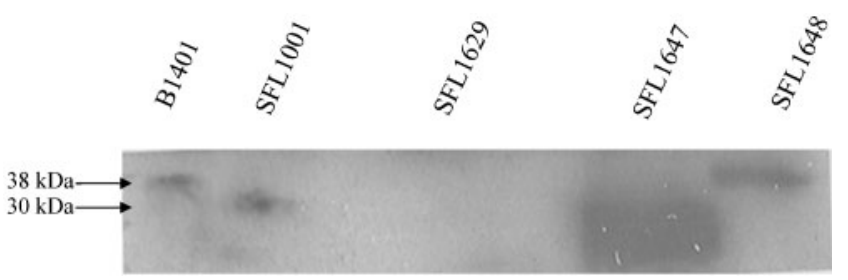

Fig. 2. Western blot showing the expression of RpoS. B1401, MG1655 E. coli strain expressing a full-sized RpoS protein; SFL1001, 2457T with truncated RpoS; SFL1629, SFL1001 with an insertion disrupting rpoS; SFL1647, SFL1629 complemented with the SFL1001 truncated RpoS; SFL1648, SFL1001 complemented with the MG1655 RpoS. The expected sizes for the full-sized RpoS and 2457T truncated RpoS are 38 and $30 \mathrm{kDa}$, respectively. Approximate band sizes were determined from the closest protein ladder bands of the Fermentas prestained PageRuler.

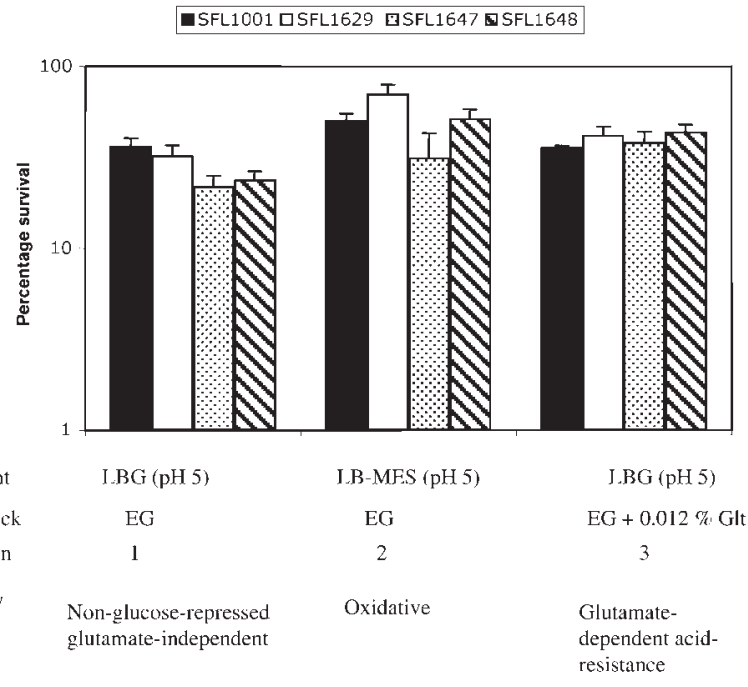

Fig. 3. Effect of RpoS disruption in S. flexneri 2457T strain SFL1001 on acid-challenge survival. Cells were grown overnight in the media indicated and diluted 1:1000 into the acid-challenge media; survival is stated as a percentage of the inoculum. Results are the mean \pm SEM of four experiments. Glt, glutamate.

using a rapid semi-quantitative catalase assay, in which an RpoS mutant will display a reduced level of bubbling, although some bubbling will still be observed as the cell possesses a second hydrogen peroxidase (HPI) that is not regulated by RpoS (Subbarayan \& Sarkar, 2004). E. coli MG1655, which possesses a full-sized (330 aa) RpoS, had a rapid, vigorous bubbling phenotype, while $S$. flexneri 2457T, which contains a truncated (255 aa) RpoS, displayed reduced oxygen production, with slow bubbling observed. Furthermore, the rpoS mutant SFL1629 was only able to slowly decompose the $\mathrm{H}_{2} \mathrm{O}_{2}$, with sluggish bubbling observed after $30 \mathrm{~s}$. SFL1647, the 2457T truncated-rpoScomplemented strain, was also severely attenuated in catalase activity, displaying bubbling similar to that observed for SFL1629, while the E. coli full-sized-rpoScomplemented S. flexneri strain produced vigorous bubbling, similar to that observed for MG1655. Therefore, the reduction in SFL1001 catalase activity in comparison to the catalase activity displayed by the full-sized E. coli RpoS suggests that the truncated RpoS is reduced in its function.

\section{Contribution of GadB and GadC to SFL1001 acid resistance}

As the GDAR pathway has been so well characterized, it seemed prudent to determine whether the unusual resistance behaviour observed in SFL1001 LBG (pH 5) cultures acid-shocked in EG ( $\mathrm{pH} 2.5$ ) had any link to the key components of the glutamate decarboxylase system. It seems unlikely that the GDAR pathway is functional under these conditions, as the pathway has an absolute requirement for glutamate to be present in the acid-shock 
medium. Disruption mutants of $\operatorname{gadB}$ and $\operatorname{gadC}$ were produced in SFL1001, and acid-resistance assays were performed on all strains, as shown in Fig. 4 . Both the gadB and gadC mutants displayed a change in their GDAR phenotype. Both strains' survival in EG $(\mathrm{pH} 2.5)$, with $0.012 \%$ glutamate added (condition 3, Fig. 4a, b), were significantly reduced in comparison to SFL1001 $(t$ tests, $P<0.01)$. Complementation of each gene disruption was sufficient to restore the acid resistance. There was no change to resistance levels of the oxidative pathway (condition 2, Fig. 4a, b), as expected, or in the unusual resistance phenotype for the gadB and gadC mutants (condition 1, Fig. 4a, b), suggesting that the key components of the GDAR pathway are not contributing

(a)

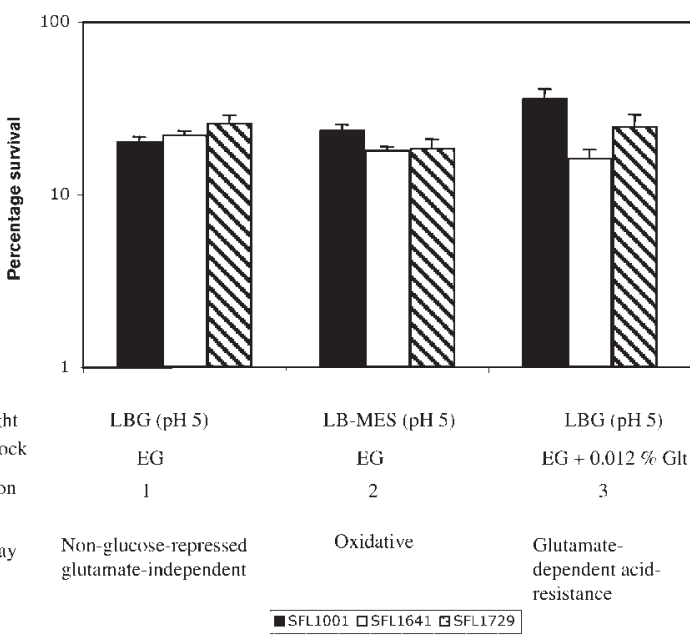

(b)

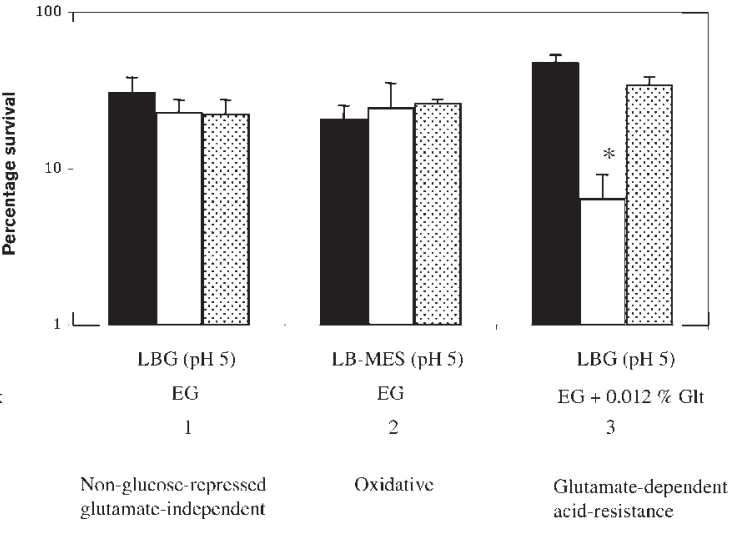

Fig. 4. (a) Acid resistance of SFL1001, SFL1650 (gadB mutant) and SFL1723 (gadB, complemented). (b) Acid resistance of SFL1001, SFL1641 (gadC mutant) and SFL1729 (gadC, complemented). Cells were grown overnight in the media indicated and diluted $1: 1000$ into the acid-challenge media; survival is stated as a percentage of the inoculum. Results are the mean \pm SEM of three experiments. SFL1650 and SFL1641 displayed significantly reduced acid resistance under condition 3 , when compared to SFL1001 by $t$ test $(P<0.01)$. Glt, glutamate. to the glutamate-independent non-glucose-repressed resistance observed in SFL1001. Interestingly, this resistance, which is observed in LBG ( $\mathrm{pH}$ 5) cultures shocked in EG $(\mathrm{pH} 2.5)$, did not prevent the loss of acid resistance for gadB and $\operatorname{gadC}$ mutants in LBG (pH 5) challenged in EG ( $\mathrm{pH} 2.5$ ) containing $0.012 \%$ glutamate (condition 3, Fig. $4 a, b)$.

\section{DISCUSSION}

The acid-resistance phenotype appears to be very important in intestinal pathogens. Bacteria must survive exposure to gastric acid in the stomach and volatile fatty acids produced within the intestine (Giannella et al., 1972). This ability to survive acidic conditions encountered during passage through the host is most likely a contributing factor to the very low infectious dose of S. flexneri. S. flexneri $2457 \mathrm{~T}$ has been widely studied in virulence assays and challenge work, and is known for its virulent phenotype; however, it has never been examined from an acid-resistance perspective (Wei et al., 2003). This study detected two acid-resistance systems, the GDAR and oxidative systems, which have been characterized elsewhere in S. flexneri 3136 (Lin et al., 1995).

The GDAR system observed in S. flexneri 2457T behaved the same as the GDAR pathway of S. flexneri 3136 (Lin et al., 1995); the pathway was acid induced, cultures requiring growth at $\mathrm{pH} 5$ to generate measurable acid resistance in acidified EG supplemented with $0.012 \%$ glutamate. Likewise, the pathway showed anaerobic induction, with higher resistance observed for cultures grown under conditions of limited oxygen availability. Additionally, the GDAR pathway was capable of operating in cells grown in minimal media at $\mathrm{pH} 5$, demonstrating that the system has no requirements for complex media during overnight growth.

The oxidative system was also present in S. flexneri $2457 \mathrm{~T}$. Cultures grown in MES-buffered LB overnight were capable of surviving exposure to acidified EG in the absence of glutamate. The pathway did not operate in cells grown without oxygenation, and showed an absolute requirement for overnight growth in complex media, as also reported for S. flexneri 3136 (Lin et al., 1995). Some properties reported for the oxidative pathway of E. coli were not detected in this study (Castanie-Cornet et al., 1999). It has been reported for E. coli that the oxidative pathway does not require acid induction by overnight growth at $\mathrm{pH} 5$ to induce resistance, but that the lack of resistance observed during growth at $\mathrm{pH} 8$ is due to the presence of a secreted inhibitor (CastanieCornet et al., 1999). The lack of resistance at $\mathrm{pH} 8$ for $S$. flexneri $2457 \mathrm{~T}$ was not due to the presence of an inhibitor, but instead seems simply to be due to a requirement for acidic growth in order to induce the pathway. Furthermore, glutamate activation was not observed, as the addition of $5.9 \mathrm{mM}$ glutamate to $\mathrm{pH} 8$ cells did not generate any oxidative resistance. 
In contrast to the behaviour reported for both E. coli and $S$. flexneri 3136, it was determined that $2457 \mathrm{~T}$ is capable of displaying resistance when grown in LBG at $\mathrm{pH} 5$ and acid-shocked in EG at $\mathrm{pH} 2.5$ without glutamate supplementation. According to the literature, these acid-shock conditions are effectively a negative control for both the GDAR and oxidative pathways (Castanie-Cornet et al., 1999; Lin et al., 1995, 1996). The GDAR system should not be able to operate under these conditions, as there is no glutamate present in the acidified EG. Likewise, the oxidative system should be repressed by the presence of glucose in the overnight growth medium. When the test cultures were grown in LBG ( $\mathrm{pH} 5$ ) without oxygenation there was a substantial drop in resistance, suggesting that this glutamate-independent, non-glucose-repressed pathway (AR4) is induced by oxidative growth. Interestingly, this pathway was also recorded, but not discussed, by de Jonge et al. (2003). In their case, E. coli O157 grown in LBG $(\mathrm{pH} 5)$ showed substantial survival in EG ( $\mathrm{pH} 2.5)$. However, no explanation was given for this unusual resistance. Whether this pathway is novel for S. flexneri 2457T and certain E. coli strains, or if it is the known oxidative pathway functioning in the presence of glucose, remains unclear. To further clarify this unusual acidresistance phenotype, mutants of S. flexneri $2457 \mathrm{~T}$ were constructed in genes known to be important to the oxidative and GDAR pathways, respectively.

As mentioned above, AR4 appears to be induced by oxidative growth. Consequently, to explore whether this resistance is from the known oxidative pathway which is not being repressed by glucose as expected, a disruption was constructed in the rpoS gene. RpoS is considered essential for the oxidative pathway to operate, and although also involved in the GDAR pathway, it is not essential, as resistance can still be detected from the GDAR system in an rpoS mutant in E. coli, and resistance is still present in S. flexneri 3136 at pH 5 (Castanie-Cornet et al., 1999; Lin et al., 1995; Small et al., 1994). The S. flexneri 2457T rpoS mutant did not show a reduction in the nonglucose-repressed, glutamate-independent acid resistance observed for LBG ( $\mathrm{pH}$ 5) cultures. However, there was also no defect or significant reduction in either the GDAR or the oxidative pathway for the rpoS knockout. It appears that the rpoS gene does not play an essential role in the regulation of any acid-resistance pathway in S. flexneri 2457T. Interestingly, this may be connected to the observation that $2457 \mathrm{~T}$ possesses a truncated RpoS protein, caused by a frameshift mutation within the gene.

This study has shown by Western blot analysis that the RpoS protein missing the last 89 amino acids is still produced in $2457 \mathrm{~T}$, but it is unclear whether it is still functional. The truncation means that the protein is missing the end of the fourth domain of the conserved $\sigma^{70}$ family structure, which is involved in -35 promoter recognition of target genes (Lonetto et al., 1992). The loss of the fourth-domain-encoding region of RpoS in E. coli is sufficient to render the protein effectively inactive
(Ohnuma et al., 2000; Subbarayan \& Sarkar, 2004). In an attempt to determine whether the 2457T RpoS is functional, a catalase assay was performed. S. flexneri $2457 \mathrm{~T}$ clearly had reduced catalase activity when compared to E. coli MG1655, which carries a full-sized RpoS. This activity was also reduced in the rpoS disruption mutant SFL1629 and in the rpoS complemented strain SFL1647, carrying the 2457T rpoS gene. It was possible to create strong catalase activity in $S$. flexneri $2457 \mathrm{~T}$ by expressing the full-length E. coli RpoS in SFL1648. These results seem to suggest that the truncated $S$. flexneri RpoS protein is at least reduced in its activity, although further research is required to confirm this.

In order to clarify any possible role of the GDAR system in AR4 resistance, disruptions were also constructed in the $\operatorname{gadB}$ and gadC genes. GadB is a glutamate decarboxylase, responsible for the conversion of intracellular glutamate to GABA. A non-polar mutation was constructed in $g a d B$ to maintain expression of $\mathrm{gadC}$, which lies downstream from gadB. The gadB knockout showed a significant reduction in GDAR, but resistance was not reduced sufficiently for it to be classified as acid sensitive ( $<10 \%$ survival). This is most likely due to the presence of the functional glutamate decarboxylase isoform gadA, which is located elsewhere in the chromosome. Castanie-Cornet et al. (1999) have demonstrated that in E. coli K12 only one Gad isoform is required for measurable acid resistance. Conversely, Waterman \& Small (2003) have shown that a gadB mutant in S. flexneri M25-8A is completely attenuated in the GDAR pathway. The gadB disruption had no effect on the AR4 resistance in S. flexneri 2457T. The complementation of the $g a d B$ mutant with a plasmid-based copy of $g a d B$ was sufficient to restore the reduced acid-resistance levels for the GDAR pathway, demonstrating that the non-polar mutation had been successful in maintaining the downstream gadC expression.

A gadC disruption was also constructed for $S$. flexneri 2457T. As expected, this strain became acid-sensitive for the GDAR pathway and gadC plasmid complementation restored the acid resistance. However, the gadC mutant was still capable of surviving exposure to EG ( $\mathrm{pH} 2.5)$ after growth in LBG ( $\mathrm{pH} 5$ ). Thus, it seems that this unusual acid resistance is perfectly operational when the GDAR pathway has been disabled and does not seem to be linked to this system. Interestingly, AR4 resistance did not rescue the $\operatorname{gadB}$ or gadC mutants, which had been rendered deficient or reduced in acid resistance under growth conditions that favour the GDAR system. The AR4 resistance phenotype could be detected when the $\operatorname{gadB}$ and gadC mutants are grown in LBG ( $\mathrm{pH} 5)$ media and shocked in EG at $\mathrm{pH} 2.5$. However, when the cells were grown as above and acid-shocked in EG ( $\mathrm{pH} 2.5)$ containing glutamate, the pathway did not appear to contribute any resistance, since if this was the case, the $\operatorname{gadB}$ - and gadC-related reductions in acid resistance would not have been observed. It may be possible that this unusual pathway is in some way repressed by glutamate. 
It still remains unclear whether the oxidative pathway is contributing to this novel resistance in a non-glucoserepressible manner. It was hoped that the previously reported absolute dependence of the oxidative pathway on RpoS could be used to determine whether the oxidative system is responsible for this non-glucose-repressible, glutamate-independent resistance. In contrast, it appears that RpoS is not important in the S. flexneri 2457T acidresistance phenotype. RpoS, the $\sigma^{S}$ subunit of RNA polymerase, is strongly induced on entry into the stationary phase and is the main regulator of the stress response in $E$. coli (Weber et al., 2005). The loss of RpoS from E. coli strains, both laboratory and natural, is quite common (King et al., 2006). It appears that loss of RpoS can actually convey a selective advantage for $E$. coli, in which rpoS deficiency may be advantageous under conditions of nutrient deprivation, may increase amino acid scavenging ability in stationary-phase cells, and even give cells a competitive advantage in mouse colonization experiments (Atlung et al., 2002; Finkel \& Kolter, 1999; Zinser \& Kolter, 1999). In this case, the S. flexneri $2457 \mathrm{~T}$ strain SFL1001 has not lost expression of rpoS, but instead produces a truncated protein with reduced activity, as demonstrated in a catalase assay. The maintenance of acid-resistance levels for the rpoS mutant of $S$. flexneri 2457T suggests that the acid-resistance pathways of the strain are able to operate independently of RpoS. All earlier reports on the loss of RpoS in E. coli and S. flexneri have shown that the oxidative pathway is consequently reduced in acid resistance, making this the first study to demonstrate RpoS independence for this pathway (Castanie-Cornet et al. 1999; Notley-McRobb, 2002; Price et al., 2000).

The acid resistance of $2457 \mathrm{~T}$ includes an unusual acidresistance phenotype. This phenotype is not related to the operation of the GDAR, as it does not display a dependence on glutamate in the acid-shock medium or require GadB and GadC. It is not clear whether this resistance is the oxidative pathway operating in the presence of glucose, or if an entirely independent pathway is present. Little is known about the oxidative pathway of S. flexneri; however, in a recent study a fur mutant of $S$. flexneri SA100 was found to be defective in the oxidative pathway (Oglesby et al., 2005). It would be interesting to determine if a fur mutant of $S$. flexneri 2457T would also be defective in oxidative acid resistance and, furthermore, determine if disruption of oxidative resistance also eradicates the novel non-glucose-repressed, glutamate-independent acid resistance.

This study is believed to be the first report on the stationary-phase acid-resistance pathways of $S$. flexneri 2457T. Two acid-resistance systems, the GDAR and the oxidative pathways, reported elsewhere for E. coli and $S$. flexneri 3136, were both detected in the S. flexneri $2457 \mathrm{~T}$ strain SFL1001. Furthermore, SFL1001 cells grown overnight under fermentative growth conditions and acidshocked in minimal medium in the absence of glutamate, an acid test often described as a negative control for both pathways, were capable of surviving acid challenge. It is unclear whether this resistance is due to the oxidative pathway operating in a non-glucose-repressible manner, or if a novel pathway is present in S. flexneri 2457T. The construction of $\operatorname{gadB}$ and gadC mutants ruled out any contribution by the GDAR pathway, whilst further characterizing the GDAR properties of S. flexneri $2457 \mathrm{~T}$. Interestingly, study of the role of rpos in the oxidative pathway and the unusual acid-resistance phenotype revealed that the frameshift present in the rpoS gene results in expression of a truncated RpoS protein, which may be reduced in activity and is not essential for the acidresistance phenotype of S. flexneri 2457T.

\section{ACKNOWLEDGEMENTS}

We wish to thank Professor T. J. Silhavy's laboratory, Princeton University, for providing us with the RpoS antibody for Western blotting.

\section{REFERENCES}

Atlung, T., Nielsen, H. V. \& Hansen, F. G. (2002). Characterisation of the allelic variation in the rpoS gene in thirteen K12 and six other nonpathogenic Escherichia coli strains. Mol Genet Genomics 266, 873-881.

Bhagwat, A. A. \& Bhagwat, M. (2004). Comparative analysis of transcriptional regulatory elements of glutamate-dependent acidresistance systems of Shigella flexneri and Escherichia coli O157:H7. FEMS Microbiol Lett 234, 139-147.

Castanie-Cornet, M. P. \& Foster, J. (2001). Escherichia coli acid resistance: cAMP receptor protein and a 20 bp cis-acting sequence control $\mathrm{pH}$ and stationary phase expression of the gadA and $\mathrm{gadBC}$ glutamate decarboxylase genes. Microbiology 147, 709-715.

Castanie-Cornet, M. P., Penfound, T., Smith, D., Elliott, J. \& Foster, J. (1999). Control of acid resistance in Escherichia coli. J Bacteriol 181, 3525-3535.

Datsenko, K. A. \& Wanner, B. L. (2000). One-step inactivation of chromosomal genes in Escherichia coli K-12 using PCR products. Proc Natl Acad Sci U S A 97, 6640-6645.

de Jonge, R., Ritmeester, W. S. \& van Leusden, F. M. (2003). Adaptive responses of Salmonella enterica serovar Typhimurium DT104 and other S. Typhimurium strains and Escherichia coli O157 to low pH environments. J Appl Microbiol 94, 625-632.

Finkel, S. E. \& Kolter, R. (1999). Evolution of microbial diversity during prolonged starvation. Proc Natl Acad Sci U S A 96, 4023-4027.

Giannella, R. A., Broitman, S. A. \& Zamcheck, N. (1972). Gastric acid barrier to ingested microorganisms in man: studies in vivo and in vitro. Gut 13, 251-256.

Gorden, J. \& Small, P. L. C. (1993). Acid resistance in enteric bacteria. Infect Immun 61, 364-367.

Hersh, B. M., Farooq, F., Barstad, D., Blankenhorn, D. \& Slonczewski, J. L. (1996). A glutamate-dependent acid resistance gene in Escherichia coli. J Bacteriol 178, 3978-3981.

Hommais, F., Krin, E., Coppee, J. Y., Lacroix, C., Yeramian, E., Danchin, A. \& Bertin, P. (2004). GadE (YhiE): a novel activator involved in the response to acid environment in Escherichia coli. Microbiology 150, 61-72.

Huan, P. T., Taylor, R., Lindberg, A. A. \& Verma, N. K. (1995). Immunogenicity of the Shigella flexneri serotype Y(SFL124) vaccine 
strain expressing cloned glucosyl transferase gene of converting bacteriophage SfX. Microbiol Immunol 39, 467-472.

King, T., Seeto, S. \& Ferenci, T. (2006). Genotype-by-environment interactions influencing the emergence of rpos mutations in Escherichia coli populations. Genetics 172, 2071-2079.

Lin, J., Soo Lee, I., Frey, J., Slonczewski, J. L. \& Foster, J. (1995). Comparative analysis of extreme acid survival in Salmonella typhimurium, Shigella flexneri and Escherichia coli. J Bacteriol 177, 4097-4104.

Lin, J., Smith, M., Chapin, K., Suk Baik, H., Bennett, G. \& Foster, J. (1996). Mechanisms of acid resistance in enterohemorrhagic Escherichia coli. Appl Environ Microbiol 62, 3094-3100.

Lonetto, M., Gribskov, M. \& Gross, C. A. (1992). The $\sigma^{70}$ family: sequence conservation and evolutionary relationships. J Bacteriol 174, 3843-3849.

Masuda, N. \& Church, G. (2003). Regulatory network of acid resistance genes in Escherichia coli. Mol Microbiol 48, 699-712.

Miller, V. L. \& Mekalanos, J. (1988). A novel suicide vector and its use in construction of insertion mutations: osmoregulation of outer membrane proteins and virulence determinants in Vibrio cholerae requires toxR. J Bacteriol 170, 2575-2583.

Notley-McRobb, L., King, T. \& Ferenci, T. (2002). rpoS mutations and loss of general stress resistance in Escherichia coli populations as a consequence of conflict between competing stress responses. J Bacteriol 184, 806-811.

Oglesby, A. G., Murphy, E. R., lyer, V. R. \& Payne, S. M. (2005). Fur regulates acid resistance in Shigella flexneri via RyhB and ydeP. Mol Microbiol 58, 1354-1367.

Ohnuma, M., Fujita, N., Ishihama, A., Tanaka, K. \& Takahashi, H. (2000). A carboxy-terminal 16-amino-acid region of $\sigma^{38}$ of Escherichia coli is important for transcription under high-salt conditions and sigma activities in vivo. J Bacteriol 182, 4628-4631.

Price, S. B., Cheng, C. M., Kaspar, C. W., Wright, J. C., DeGraves, F. J., Penfound, T. A., Castanie-Cornet, M. P. \& Foster,, J. W. (2000). Role of rpoS in acid resistance and fecal shedding of Escherichia coli O157:H7. Appl Environ Microbiol 66, 632-637.

Richard, H. \& Foster, J. W. (2004). Escherichia coli glutamate- and arginine-dependent acid resistance systems increase internal $\mathrm{pH}$ and reverse transmembrane potential. J Bacteriol 186, 6032-6041.
Ruiz, N. \& Silhavy, T. J. (2003). Constitutive activation of the Escherichia coli Pho regulon upregulates rpoS translation in an Hfqdependent fashion. J Bacteriol 185, 5984-5992.

Sambrook, J. \& Russell, D. W. (2001). Molecular Cloning: a Laboratory Manual, 2nd edn. Cold Spring Harbor, NY: Cold Spring Harbor Laboratory.

Small, P., Blankenhorn, D., Welty, D., Zinser, E. \& Slonczewski, J. L. (1994). Acid and base resistance in Escherichia coli and Shigella flexneri: role of rpoS and growth $\mathrm{pH}$. J Bacteriol 176, 1729-1737.

Subbarayan, P. R. \& Sarkar, M. (2004). A comparative study of variation in codon 33 of the rpoS gene in Escherichia coli K12 stocks: implications for the synthesis of $\sigma^{\mathrm{s}}$. Mol Genet Genomics 270, 533-538.

Vogel, H. J. \& Bonner, D. M. (1956). Acetylornithinase of Escherichia coli: partial purification and some properties. J Biol Chem 218, 97-106.

Waterman, S. R. \& Small, P. (1996). Identification of $\sigma^{\mathrm{s}}$-dependent genes associated with the stationary-phase acid-resistance phenotype of Shigella flexneri. Mol Microbiol 21, 925-940.

Waterman, S. R. \& Small, P. L. (2003). Identification of the promoter regions and $\sigma^{\text {s }}$-dependent regulation of the gadA and $\operatorname{gadBC}$ genes associated with glutamate-dependent acid resistance in Shigella flexneri. FEMS Microbiol Lett 225, 155-160.

Weber, H., Polen, T., Heuveling, J., Wendisch, V. F. \& Hengge, R. (2005). Genome-wide analysis of the general stress response network in Escherichia coli: $\sigma^{\mathrm{s}}$-dependent genes, promoters and sigma factor selectivity. J Bacteriol 187, 1591-1603.

Wei, J., Goldberg, M. B., Burland, V.,, Venkatesan, M. M., Deng, W., Fournier, G., Mayhew, G. F., Plunkett, G., 3rd, Rose, D. J. \& other authors (2003). Complete genomic sequence and comparative genomics of Shigella flexneri serotype 2a strain 2457T. Infect Immun 71, 2775-2786.

Zinser, E. R. \& Kolter, R. (1999). Mutations enhancing amino acid catabolism confer a growth advantage in stationary phase. J Bacteriol 181, 5800-5807.

Edited by: D. J. Jamieson 